\title{
Volker Gerhardt
}

\section{Politische Ordnung als Bedingung religiöser Freiheit}

In: Nida-Rümelin, Julian / Daniels, Detlef von / Wloka, Nicole (Hrsg.): Internationale Gerechtigkeit und institutionelle Verantwortung. - ISBN: 978-3-11-061586-9. - Berlin: De Gruyter, 2019.

S. $385-392$

(Forschungsberichte / Interdisziplinäre Arbeitsgruppen, Berlin-Brandenburgische Akademie der Wissenschaften ; 41) 


\section{Volker Gerhardt}

\section{Politische Ordnung als Bedingung religiöser Freiheit}

Es wäre mir unmöglich, auch nur den Versuch zu machen, den dichten Zusammenhang von Wissen und Glauben und damit auch den von Politik und Religion in einem Kurzvortrag zum Thema zu machen, wenn ich anderswo dazu nicht schon mehr gesagt hätte (Gerhardt 2015, 2017). So aber kann ich mir erlauben, mit der Geschichte großzügig umzugehen und kurzerhand zu systematischen Fragen zu kommen, die es ermöglichen, zu einem in nahezu allen Weltgegenden unzureichend oder gar nicht gelösten praktischen Problem einen Vorschlag zu machen, der eine elementare Bedingung von Verständigung und Frieden auf der Welt betrifft. Darin liegt keine Geringschätzung der sozialen Gegensätze oder des Verlangens nach Wohlstand und äußerer Sicherheit, allein schon deshalb nicht, weil auch hier die Religionen einen Grund, einen Anlass oder auch einen Vorwand für ihren Beitrag zum Leben der Menschen haben.

\section{1}

Nach einer Vorlesung, in der ich auf vorantike Stationen des Nachdenkens über Technik hingewiesen hatte, wurde ich gefragt, warum ich bei der Behandlung des Alten Testaments zwar die mühevolle Feldarbeit durch Adam, Kain und ihre Nachkommen, die Arche Noah, die über ein Jahr in Anspruch nehmende kosmetische Vorbereitung der Esther auf ihre Hochzeit mit Nebukadnezar, die Klage Hiobs über den Frevel durch den das Erdreich durchwühlenden Bergbau sowie den aufwendigen Palastbau des Königs Salomon behandle - aber nicht den Turmbau zu Babel, der doch zum Sinnbild technischer Überheblichkeit des Menschen geworden ist.

Nach erneuter Lektüre der kurzen Passage in Genesis 11 konnte ich nur sagen, dass es dort nicht, wie man landläufig meint, um die Bestrafung der technischen Hybris des Menschen, sondern um seinen Ungehorsam gegenüber dem Befehl Gottes geht: Noah und seine Söhne hatten nach überstandener Flut den göttlichen Auftrag erhalten, sich in alle Länder auszubreiten und sich dort fruchtbar zu mehren. Doch sie waren nur in die üppig ausgestattete mesopotamische Ebene hinabgestiegen und schickten sich an, mit dem Wolkenkratzer ein weithin sichtbares Zeichen ihrer Sesshaftigkeit zu geben.

Ә OpenAccess. (c) 2019 Volker Gerhardt, publiziert von De Gruyter. (cc))BY-Nc-SA Dieses Werk ist lizenziert unter der Creative Commons Attribution-NonCommercial-ShareAlike 4.0 Lizenz. 
Also diente die Sprachverwirrung dem Ziel, die Menschen auseinanderzutreiben, so dass sie sich in ihrem wechselseitigen Nichtverstehen weltweit verteilen und in ihrer jeweiligen Sprache ihre eigenen Kulturen gründen würden.

Es wäre blasphemisch, darüber zu spekulieren, was die Absicht Gottes bei diesem Migrationsbefehl gewesen sein könnte. Aber es dürfte auch einen Gott nicht überrascht haben, dass die Menschen an den Orten ihrer neugefunden Aufenthalte mit der Zeit auch ihre eigenen Götter verehrten. Die wechselvolle Geschichte des jüdischen Volkes von Abraham über Joseph und die Könige zeigt, dass nicht nur der eigene Glauben, sondern auch die Wahrnehmung des abweichenden Glaubens der anderen eine Konstante, ja sogar ein Prinzip der Treue Gottes zu seinem Volk gewesen ist.

Doch ganz gleich, was sich Gott bei der Zulassung so vieler Religionen allein im afro-eurasischen Raum des Vorderen Orients gedacht haben mag: Die Vielfalt der Religion ist ein historischer Tatbestand, der wesentlich zur Kulturgeschichte der Menschheit gehört: Die Gemeinschaften der Menschen wussten von ihren religiösen Differenzen, haben sie gepflegt und sich dabei keineswegs nur wechselseitig voneinander abgegrenzt! Sie haben sich vielmehr gerade in religiösen Dingen aufmerksam beobachtet und haben voneinander gelernt. Sie waren besonders daran interessiert, welche Götter und welche Riten jeweils bei den anderen erfolgreich waren. Die Neigung, die Erfolg verheißenden rituellen Praktiken zu übernehmen, ist vielfach belegt. Herodot führt vor, wie sehr dies auch für die Griechen des fünften vorchristlichen Jahrhunderts gegolten hat.

Damit bin ich bei meinem ersten Punkt: Die Vielfalt der Religionen, mit ihren zahlreichen Gemeinsamkeiten und partiellen Gegensätzen, ist keine neue Erscheinung der sogenannten globalisierten Welt, sondern sie ist das seit Menschengedenken vorgegebene Element des schon immer im Weltmaßstab erfahrenen religiösen Lebens. Das relativ Neue besteht darin, dass territoriale Grenzen nicht länger einen (wenigstens erhofften) Schutz vor den Religionen der anderen bieten und dass auf der inzwischen restlos besiedelten und damit klein gewordenen Oberfläche der Erde sich keine Religion die Freiheit herausnehmen kann, auch in politischer Absicht über die Bewohner eines Territoriums zu bestimmen. Jede - auch noch so abgelegene - politische Herrschaft muss grundsätzlich dem Lebensrecht aller Menschen genügen.

\section{2}

Soweit wir sehen können, gehen die zivilisatorisch wirksamen Religionen in ihrer Entstehung und Entwicklung von dem zu ihrer Zeit verfügbaren Wissen aus. Das Wissen legt auch den Grund für die auf das Göttliche gerichteten Erwartungen. 
Insofern haben sie alle einen rationalen Kern, der auch darin sichtbar bleibt, dass Wissen dem Glauben die propositionale Struktur sowie die gleichen grammatischen und semantischen Regeln vorgibt.

Der Glauben wird lange Zeit gar nicht eindeutig vom Wissen unterschieden. Aber es ist erkennbar, dass zur Zeit des Aufkommens von schriftlich überlieferten und versicherten Religionen bereits erste Praktiken des technischen, mathematischen, astronomischen, geographischen und medizinischen Wissens zur Verfügung stehen, so dass man genügend Erfahrung damit haben kann, dass alles Wissen von den durch menschliches Tun direkt in Gang zu setzenden und durch Berechnungen vorhersehbaren Vorgängen nicht ausreicht, um allein die Wissbegierde, geschweige denn die persönlichen und allgemein menschlichen Erwartungen, die mit dem Leben und Handeln verbunden sind, zu befriedigen. Man hatte schon hinreichend Erfahrungen mit dem Wissen, um sicher sein zu können, dass es nicht in der Lage ist, den Menschen wenigstens in den entscheidenden Lebensfragen Antworten zu geben oder auch nur Trost zu spenden.

Es reicht vor allem nicht mit Blick auf das, was jeder über seine persönliche Zukunft, über den Verlauf von Naturkatastrophen, den Ausgang folgenschwerer Geschäfte oder über den Ausgang von Kriegen wissen möchte. So sollte und konnte die nach Art eines Wissens von ahnungsvollen Propheten, hohen Priestern oder kundigen Schriftgelehrten verkündete Gewissheit Abhilfe schaffen, um wenigstens Zuversicht zu verbreiten.

Erst unter dem Einfluss eines methodischen Nachdenkens durch die Philosophen des fünften und vierten vorchristlichen Jahrhunderts wurden Wissen und Glauben kategorial getrennt, und - spätestens mit dem Wirken des Apostels Paulus - auch in die christliche Überlieferung übernommen.

Heute tun wir so, als stünden sich Wissen und Glauben wie zwei gegensätzliche Einstellungen des Menschen zu sich und seiner Welt gegenüber. Und so vielfältig die in Philosophie, Theologie und Wissenschaftstheorie verhandelten Unterscheidungen auch sind (vgl. Kemmerling 2017): ${ }^{1}$ Sicher ist, dass Wissen und Glauben nicht nur auf das Engste zusammengehören, sondern dass sie sich wechselseitig fordern!

Das ist allein daran zu sehen, dass bereits der ernsthafte pragmatische Einsatz des Wissens einen Glauben an das Wissen voraussetzt und dass dort, wo wir uns mit dem sich ja in allen Lebenslagen rasch als unzureichend zeigenden

1 Wenn ich auf weitergehende Literaturhinweise verzichte, bedeutet das nicht, dass es keine gibt. Dass die philosophische Literatur zwischen Platon, Kant, Hegel und Nietzsche voller wichtiger Hinweise ist und im Hintergrund steht, muss ich nicht betonen. Aber es gibt auch neuere Arbeiten von Bultmann, Kutschera, Dalfert, Schulz und Petzold - vom Wittgenstein des Tractatus ganz zu schweigen. 
Wissen befassen, ein Glauben nötig ist. Auch wenn die Wissenschaftler dies nicht gerne zugeben: Bereits an der Grenze zwischen Gegenwart und Zukunft hat das Wissen dem Glauben Platz zu machen. Und es versagt völlig, wenn wir uns fragen, was das Leben - nicht nur in diesem oder jenem Verlauf, sondern überhaupt bedeutet.

Sinnfällig wird das an den jederzeit benötigten Überzeugungen in Moral, Politik und Kultur, so dass die Hauptfrage darin besteht, worin die Besonderheit des religiösen Glaubens besteht. Hier reicht es mit Blick auf die Religion nicht aus, auf den höheren Anteil des Gefühls, die größere Verbindlichkeit kultureller und familiärer Traditionen, die ästhetische Eindringlichkeit religiöser Überlieferungen sowie auf ein Vertrauen in ein göttlich (oder Gott) genanntes Ganzes des Daseins und der Welt zu verweisen. Denn es ist offensichtlich, dass auch nicht-religiöse Formen des Selbst- und Weltvertrauens ohne vergleichbare affektive, traditionale und totalisierende Annahmen über die jeweilige Ganzheit von Person, Menschheit, Geschichte und Welt nicht auskommen.

Auch das religiöse Bewusstsein beruht wesentlich darauf, wie sich das Individuum in seinem Welt- und Selbstverhältnis begreift. Um das zu qualifizieren, werden gern die Attribute des „Subjektiven“ und des „Privaten“ verwendet. Und je nach Kontext tun sie durchaus ihren Dienst:

Als „subjektiv“ gilt in der Wissenschaftssprache alles, was der „Objektivität“ der Erkenntnis gegenübersteht. Auch wenn das subjektive Erleben in Ethik und Ästhetik reich und vielfältig sein kann, ist es doch durch den Gegensatz zur Objektivität lediglich negativ bestimmt. Und das ist, so meine ich, zu wenig.

Beim Begriff des „Privaten“, der ja schon von seiner ursprünglichen Bedeutung her den „Raub“, den „Vorbehalt“ und somit die „Ausnahme“ von der Zuständigkeit der res publica bezeichnet, ist das nicht anders. In der jeweiligen Begrenzung kann es gut und richtig, ja notwendig sein, den Glauben an das Göttliche als „subjektiv“ und „privat“ zu bezeichnen. Denn das lässt viel Raum, um den religiösen Glauben nicht als zwingend für alle und jeden zu verstehen. Wer seine Gründe hat, sich als glaubensfern, ungläubig oder atheistisch zu bezeichnen, muss die Freiheit haben, in dieser Überzeugung zu leben, ohne Nötigung, auch nur seine Gründe offenzulegen.

In theologischer Dimension aber greifen beide Begriffe, der des Subjektiven wie der des Privaten, zu kurz! Hier liegt in der Individualität die angemessene Auszeichnung eines Glaubens, der sich unmittelbar dem Universellen gegenübersieht. Dafür gibt es einen guten metaphysischen Grund: Das eine zeigt sich nur im innigsten Kontrast zum anderen, ohne dazu einer weiteren Rahmenbedingung, wie Objektivität oder Publizität sie benötigen, zu bedürfen (vgl. Gerhardt 2012). 
Der Glauben an das Göttliche geht über alle subjektiven oder privaten Formen der Meinung, der Annahme, der Überzeugung, der Erwartung, der Zuversicht oder der Hoffnung hinaus, indem er niemals bloß als individuell, sondern immer auch als existenziell und zugleich als universell begriffen und gewürdigt werden muss. Dies aber, ohne einen Anspruch mit sich zu führen, andere auf denselben Weltbegriff $\mathrm{zu}$ verpflichten - andere also, die ihrerseits auch in ihrer Individualität anerkannt werden müssen, um überhaupt als Gesprächs- oder Handlungspartner infrage zu kommen. Anders als Subjektivität kann Individualität nicht durch Argumentation, schon gar nicht durch Zwang überboten oder überblendet werden. Die Individualität des Anderen wird in Korrespondenz zum eigenen Selbstverständnis zwingend vorausgesetzt.

Unabhängig davon bedarf auch jeder Glauben des Wissens, wie es in den Büchern und Lehren der tradierten Religionen bewahrt ist, wie es durch die Kulturgeschichte und die Theologie präzisiert werden kann und wie es sich durch die Erkenntnisse der Wissenschaften belehren lassen muss.

\section{3}

Nach dem Sprung in die Vorgeschichte und dem nachfolgenden Schritt zur systematischen Analyse des notwendigen Zusammenhangs zwischen den (sehr wohl zu unterscheidenden) Leistungen des Wissens und des Glaubens, wage ich einen kurzen Satz in die politische Zukunft, die nirgendwo anders als bereits in der Gegenwart beginnt.

So stark Religionen mit Wissen aufgeladen sind, so viel unschätzbare Gelehrsamkeit in ihren heiligen Büchern auch bewahrt sein mag: Sie scheinen von sich aus nicht die Kraft zu haben, sich auf ihre Gläubigen und damit auch auf sich selbst zu beschränken. Glaubensgemeinschaften glauben offenbar nicht, ihren Gläubigen den Weg zum Heil allein durch den Glauben ebnen zu können. Sie halten es vielmehr für ihre Pflicht, auch die Nicht-Gläubigen davon zu überzeugen, dass vornehmlich ihre Praxis, die Lebensform, auf die sie zu verpflichten suchen, die von ihnen offerierte Erlösung versprechen können.

Diese Überzeugung, so abwegig sie angesichts der gleichzeitig agierenden Vielzahl von Religionen auch erscheinen mag, wird man den Religionen nicht ausreden können. Sie gehört offenbar zu ihrem Selbstverständnis, so wenig Selbstvertrauen in den Gehalt ihrer Heilsbotschaft darin auch zum Ausdruck kommt. Religiöser Glauben möchte sich vor Gott und vor seinesgleichen bezeugen, so sehr die individuelle Natur des Glaubens dem auch entgegensteht. Selbst Lutheraner, die ihren Glauben im hoch individualisierten Bewusstsein des solus Christus bekennen und mit Recht die Friedfertigkeit dieses Jesus Christus ins 
Zentrum rücken, sprechen so, als sei nichts dabei, sich auf den angeblichen „Missionsbefehl“ ihres Heilands zu berufen.

Solange die Religionsgemeinschaften hier nicht selbst zu einer angemessenen Selbstbescheidung finden, muss man ihrer Missions- und Bekehrungslust Grenzen setzen! Sie müssen daran gehindert werden, die Verkündigung ihres Glaubens mit Aufdringlichkeit oder gar mit Zwang und Gewaltsamkeit zu betreiben. Dabei haben sie auch Kindern und Jugendlichen gegenüber darauf zu achten, dass deren Überzeugungen sich in der Entfaltung ihrer freien Einsicht und ihres eigenen Urteils bilden können.

Dass dies in Familien und in kleinen Gemeinschaften keine leichte Aufgabe ist, sieht man sofort. Aber hier wenigstens einen Vorsatz, vielleicht sogar eine Maxime oder ein Ethos zu begründen, ist ein elementarer Punkt in der Selbsterziehung der Religionen, die auf Dauer nur so wahrhaft überzeugte Anhänger gewinnen können. Wenn es richtig ist, dass der Glauben letztlich nur eine individuell gegründete, lediglich existenziell gültige und am Bewusstsein der Universalität des Ganzen ausgerichtete Erwartung sein kann, muss auch die Erziehung dahin individuelle Wege eröffnen.

Dazu gehört, dass sich die Glaubensgemeinschaften dem Einzelnen gegenüber nicht nach Art einer Zensurbehörde seines Wissens und seines Urteils aufspielen. Jeder muss, um nur ein Beispiel zu nennen, aufgrund seines selbsterworbenen Wissens selbst entscheiden können, ob er den Darwinismus für wissenschaftlich aufschlussreich hält oder nicht. Er muss auch, ungeachtet aller gesellschaftlichen Hilfspflichten, selbst darüber bestimmen können, wie er sterben möchte.

Im Vergleich zur Lage nach dem gescheiterten Turmbau zu Babylon ist das wahrhaft neue der gegenwärtigen Situation, dass die Menschen mit ihrem jeweiligen (schon längst nicht mehr bloß an die Sprache gebundenen) Welt- und Selbstverständnis nicht mehr voreinander ausweichen können. Es ist auch nicht mehr allein der knappe Raum auf der Oberfläche der Erde, der die Menschen aneinanderbindet. Wissenschaft, Technik, Handel, Sport und Tourismus - nicht zu vergessen: die gemeinsame Lebensgefahr, unter der sie durch ihre selbstproduzierten Waffen in jeder Minute stehen - machen die Menschheit zu einer kleinen planetaren Schicksalsgemeinschaft, die sich verbindlichen Verhaltensregeln zu unterstellen hat, wenn es sie noch in einer Weise geben soll, dass es erlaubt ist, von Menschheit zu sprechen und auf Humanität zu hoffen. Die wiederum ist es, die einen Weg zu einem von eigenen Einsichten getragenen Glauben ermöglicht, der nicht in Konflikt zu einer Politik stehen muss, die sich die Erhaltung und Entfaltung der Menschheit zum Ziel setzt.

Eine solche das einvernehmliche Nebeneinander von Religion und Politik ermöglichende Form des Lebens muss auf für beide Seiten verbindliche Regeln 
gegründet sein: Sie müssen auf Gegenseitigkeit angelegt sein, sie haben die prinzipielle Gleichheit und Freiheit eines jeden nicht nur anzuerkennen, sondern auch zu sichern. Solche Regeln können nur rechtliche sein, die politisch durchzusetzen und mit zivilen Mitteln zu wahren sind. Wenn die Religionen in der sie tragenden Vielfalt ihrer Bekenntnisse noch eine Zukunft haben wollen, haben sie die Dominanz des allgemeinen Rechts und die Ordnungsleistung der Politik anzuerkennen.

Der Verzicht auf den „Missionsbefehl“ ist hier nicht mehr als ein Beispiel für das, was von den Kirchen in einer ihre religiöse Vielfalt anerkennenden Welt erwartet werden muss. Dabei können sie sich gegenseitig ein Beispiel geben. Aber die Impulse dazu müssen aus politischen Einsichten kommen, die letztlich menschenrechtlich fundiert sein müssen. Deshalb kann in der modernen Welt keine Religion eine Zukunft haben, die das Menschenrecht nicht anerkennt und die nicht auch in ihrem Inneren jedem Gläubigen das Recht gewährt, die Entscheidung für oder gegen den Glauben selbst zu treffen.

Aus der menschenrechtlichen Verpflichtung aller Religionen folgt, dass keiner Religion der Status einer rechtsprechenden Gewalt zuerkannt werden kann. Es mag begrenzte Möglichkeiten zu einer Disziplinargerichtsbarkeit geben, wie sie in Berufsgenossenschaften, in der Wissenschaft oder im Sport üblich sind. Aber das Recht, die Freiheit eines Menschen einzuschränken, kann keiner noch so ehrwürdigen religiösen Institution zugestanden werden. Es ist die Politik, die hier die Regeln vorgibt und die Aufsicht führt.

Auch mit diesen Hinweisen gebe ich nur Beispiele für den Vorrang der politischen Ordnung für alles, was im Handeln von Religionsgemeinschaften geeignet sein könnte, die Freiheit von Mitbürgern, welchen Glaubens sie auch sind, zu gewähren. Die Politik als weltliche Macht hat die Ordnung für das gemeinschaftliche Leben vorzugeben und für deren Einhaltung zu sorgen.

\section{4}

Das alles schließt nicht aus, dass die Religionen höchst erwünschten Anteil am zivilgesellschaftlichen Leben nehmen. Sie haben im Inneren wie im Verhältnis der Staaten zueinander ihre Stimme zu erheben und sollten in allen Fragen des öffentlichen Lebens durch Abgabe ihres begründeten Urteils gegenwärtig sein, sobald und solange es um religiöse Belange geht. Doch auch hier haben sie die Freiheit des Einzelnen ernster zu nehmen als sie dies etwa in den biopolitischen Debatten der Gegenwart oder in der Aufarbeitung der Missbrauchsskandale tun.

Insgesamt ist $\mathrm{zu}$ wünschen, dass alle Religionen die Konsequenz ziehen, gemeinsam Verantwortung für das zivile Leben zu tragen. Das wissenschaftliche 
Wissen organisiert sich längst in international vernetzten Vereinigungen, die eine eigene Stimme haben und vielfältige Formen der Beratung von Gesellschaft und Politik nutzen. Die Wissenschaft erkennt längst ihre Verbindlichkeiten gegenüber der menschlichen Gemeinschaft an. Der Glauben ist dagegen im internationalen Rahmen nur partiell organisiert, wie etwa in dem auch „Weltkirchenrat“ genannten Ökumenischen Rat der Kirchen oder in der seit Jahrhunderten weltweit agierenden römischen Kurie. Hier werden verständlicherweise primär die Interessen der zugehörigen Gläubigen wahrgenommen.

Dabei darf es nicht bleiben! Es gibt weltweite technologische, wissenschaftliche und politische Entwicklungen, die den Glauben insgesamt betreffen. Darüber sollten sich die Glaubensgemeinschaften auch weltweit verständigen. In den großen Fragen der globalen Politik: Frieden, Schutz der Umwelt, Gesundheit und Ernährung, Wahrung des Rechts und namentlich Asyl, Terrorismus und Toleranz, sind sie alle betroffen. Es käme somit darauf an, dass die Religionen in ein sie alle einbindendes Gespräch miteinander finden, um in der Lage zu sein, angesichts der zahlreichen Probleme, die sie alle betreffen, mit einer Stimme zu sprechen.

Es ist ein vorrangiges weltpolitisches Ziel, dass möglichst alle Glaubensgemeinschaften in einer weltumspannenden Organisation zusammenfinden, um aktiv an der Wahrung einer Welt mitzuwirken, in der der Glauben geschützt und geachtet wird. Das könnte nach Art der UNO, also in einer ständigen Weltkonferenz der Religionen geschehen und sollte eine Chance bieten, deren eigenes Interesse gegenüber der Politik zu artikulieren. So paradox es klingt: Ein globales Forum der Glaubensgemeinschaften könnte auch einen Weg eröffnen, den Glauben von seiner Abhängigkeit von einer Politik zu befreien, die nur die Machtinteressen der jeweils Herrschenden verfolgt.

Wenn es gelänge, in Anerkennung der Notwendigkeit des Wissens und der Wissenschaft sowie im Respekt vor den Instanzen des Rechts und einer ihm verpflichteten Politik ein weltweit sichtbares Zeichen für die Freiheit des Glaubens zu setzen, wäre das wohl der wichtigste Dienst, den man dem Glauben erweisen kann.

\section{Bibliographie}

Gerhardt, Volker (2012): Öffentlichkeit. Die politische Form des Bewusstseins. München: Beck. Gerhardt, Volker (2015): Der Sinn des Sinns. Versuch über das Göttliche. 4. Auflage. München: Beck.

Gerhardt, Volker (2017): Wissen und Glauben. Ein notwendiger Zusammenhang. 2. Auflage. Stuttgart: Reclam.

Kemmerling, Andreas (2017): Glauben. Frankfurt am Main: Klostermann. 